\title{
Is HOTAIR really involved in neuroendocrine prostate cancer differentiation?
}

\author{
Rebecca L. Mather ${ }^{1}$, Yuzhuo Wang ${ }^{2}$ \& Francesco Crea*,1 \\ ${ }^{1}$ School of Life, Health \& Chemical Sciences, The Open University, Walton Hall, Milton Keynes, MK7 6AA, UK \\ 2 Department of Experimental Therapeutics, BC Cancer Research Centre, Vancouver, British Columbia, Canada \\ *Author for correspondence: francesco.crea@open.ac.uk
}

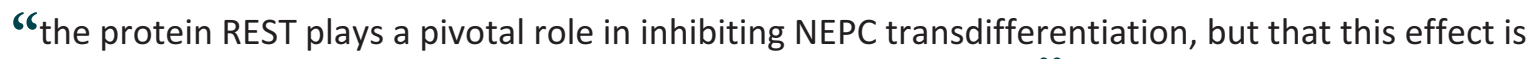
not mediated by the HOTAIR InCRNA"

First draft submitted: 6 July 2018; Accepted for publication: 9 July 2018; Published online:

21 September 2018

Keywords: Prostate cancer • long non-coding RNA • HOTAIR • REST • MIAT

Long noncoding RNAs (lncRNAs) are emerging as key players in several aspects of human pathophysiology [4]. Upon transcription, lncRNAs fold in tridimensional structures and can interact with epigenetic effectors, signalling proteins and other macromolecules, thereby influencing a diverse range of cellular phenotypes. However, due to the limited information available on lncRNA function and expression profiles, it is sometimes difficult to define their roles in specific diseases.

HOTAIR is an oncogenic lncRNA, which was first discovered for its role in breast cancer metastasis [3]. Successive studies have confirmed the oncogenic role of HOTAIR in other malignancies, including prostate cancer. As shown in Figure 1A, prostate malignancies can progress through three main stages: hormone-sensitive tumors, which are treatable by a combination of radiotherapy, surgery and hormonal therapy; castration-resistant prostate cancer (CRPC) which is resistant to conventional androgen deprivation therapy, but can be treated by more potent hormonal drugs (enzalutamide, abiraterone); neuroendocrine prostate cancer (NEPC), which is currently incurable. Clinical and experimental evidence indicates that the HOTAIR lncRNA drives the progression from androgen-sensitive to CRPC [3].

We have previously shown that the lncRNA MIAT might be involved in a further step of prostate cancer progression: the transdifferentiation from prostatic adenocarcinomas to NEPC [2]. Our analysis of clinical databases showed that MIAT expression is significantly higher in NEPC samples, compared with all other prostate cancer subtypes, and that MIAT upregulation is associated with worse prostate cancer prognosis. NEPC is the deadliest form of prostate cancer. Hence, understanding the mechanisms underpinning this transdifferentiation is of fundamental importance. For this reason, we are currently working on the functional characterization of MIAT and other lncRNAs that could be involved in NEPC.

A recent experimental paper claimed that the HOTAIR lncRNA also plays a pivotal role in NEPC transdifferentiation [1]. The authors found that HOTAIR upregulation induced the expression of some NEPC markers in prostate cancer cells, and that HOTAIR expression was inhibited by the transcriptional repressor REST. Since REST is downregulated during the transition from CRPC to NEPC, the authors propose that REST silencing triggers HOTAIR expression in NEPC cells (Figure 1A). An analysis of clinical prostate cancer samples showed that HOTAIR expression is higher in CRPC, compared with hormone sensitive malignancies. Crucially, the authors did not compare the expression of HOTAIR in NEPC versus CRPC samples.

In light of this evidence, we sought to corroborate the role of HOTAIR in NEPC transdifferentiation by analyzing our patient-derived models and a clinically available dataset (CbioPortal, 'Trento-Cornell-Broad 2016'). As shown in Figure 1B we found that, while REST is consistently downregulated in NEPC versus CRPC/adenocarcinoma samples, HOTAIR is expressed at very similar levels in the two groups. Notably, the clinical dataset confirmed our previous findings on MIAT, showing that this lncRNA is selectively upregulated in NEPC samples.

Future Medicine 
(A) Treatment resistance

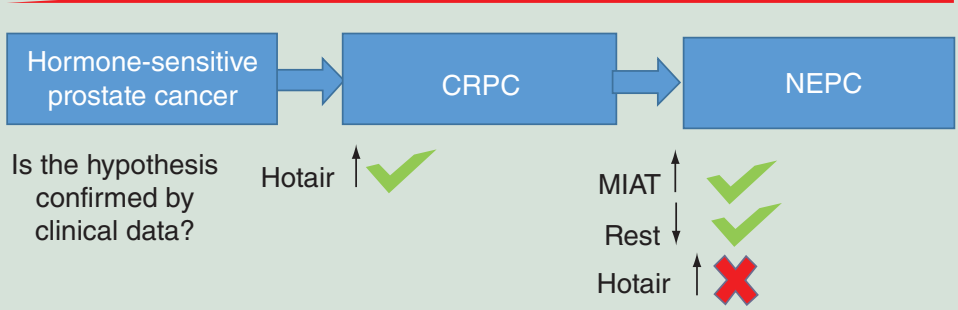

(B)
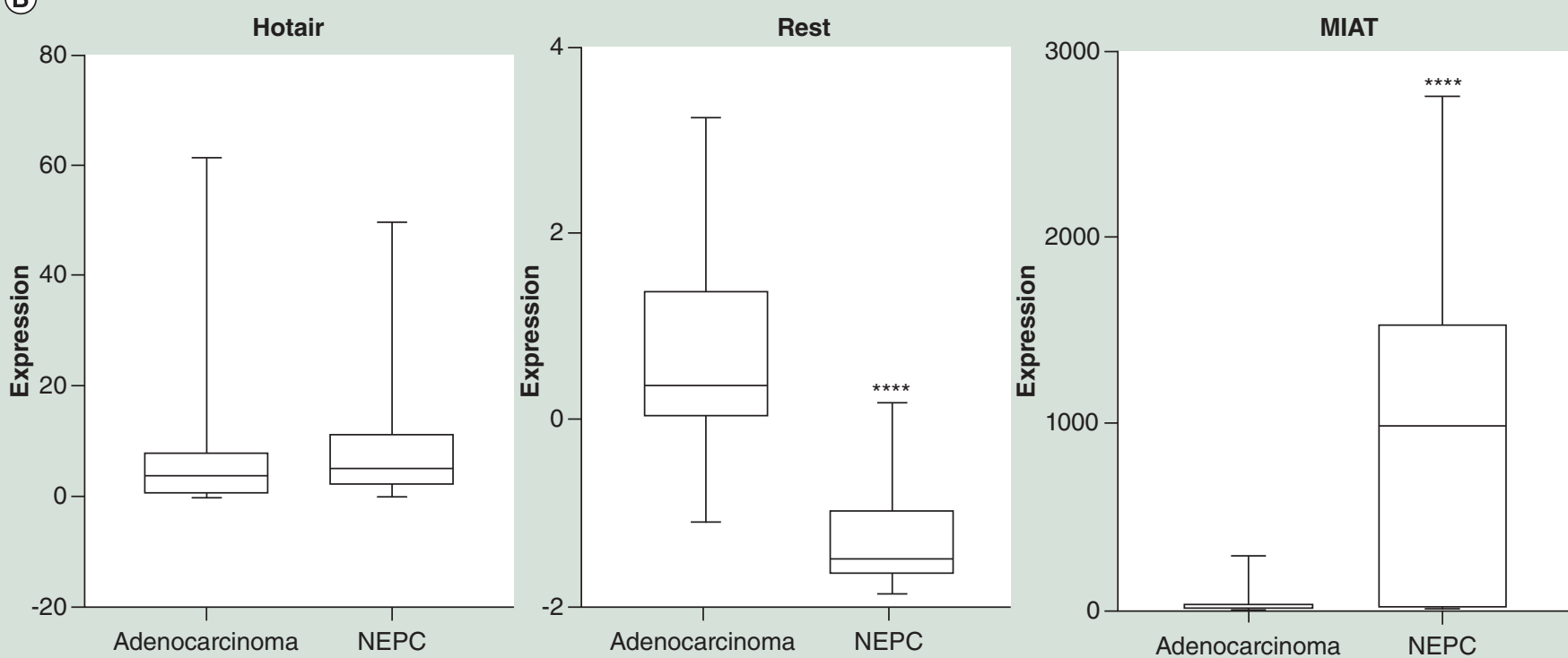

(C)
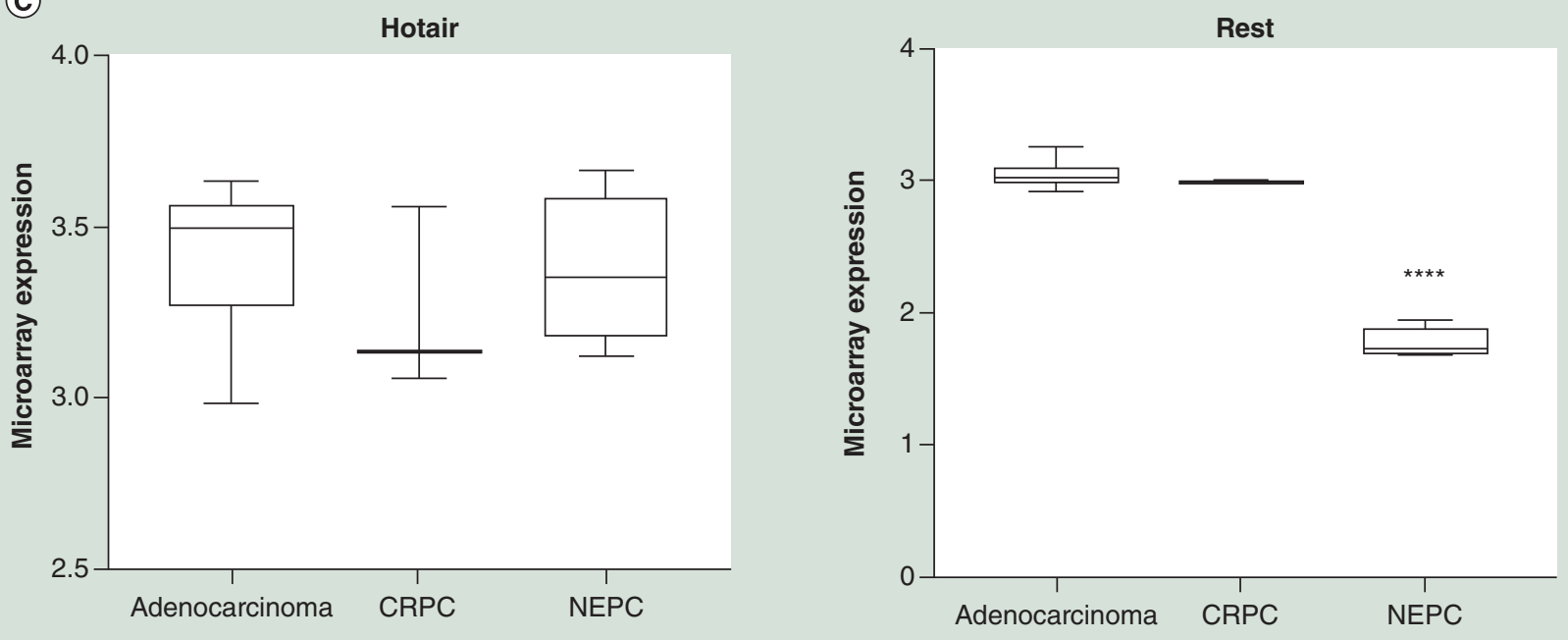

Figure 1. The role of HOTAIR, REST, and MIAT in prostate cancer progression. (A) A unified model describing the role of HOTAIR, REST, and MIAT in the three main stages of prostate cancer progression. (B) Clinical samples from patients with prostate adenocarcinoma, and neuroendocrine prostate cancer (NEPC). REST is signficantly downregulated in NEPC vs adenocarcinoma ( $<<0.0001)$. MIAT is signficantly up-regulated in NEPC vs adenocarcinoma ( $p<0.0001)$. (C) PDX models of prostate adenocarcinoma, castration resistant prostate cancer (CRPC), and neuroendocrine prostate cancer (NEPC). REST is signifiantly down-regulated in NEPC vs adenocarcinoma and CRPC $(<0.0001)$. (C) Data analysed by two-tailed $t$-test. Data analyzed by one-way analysis of variance with Tukey's post-hoc test. All data analyzed using graphpad Prism 7 software. 
Based on this evidence, we propose that the protein REST plays a pivotal role in inhibiting NEPC transdifferentiation, but that this effect is not mediated by the HOTAIR IncRNA. We believe that further studies are needed to dissect the functional role of lncRNAs in NEPC.

\section{Financial \& competing interests disclosure}

This article was partially supported by Cancer Research UK DDC project grant 22592. The authors have no other relevant affiliations or financial involvement with any organization or entity with a financial interest in or financial conflict with the subject matter or materials discussed in the manuscript apart from those disclosed.

No writing assistance was utilized in the production of this manuscript.

\section{References}

1. Chang YT, Lin TP, Tang JT et al. HOTAIR is a REST-regulated IncRNA that promotes neuroendocrine differentiation in castration resistant prostate cancer. Cancer Lett. 433, 43-52 (2018).

2. Crea F, Venalainen E, Ci X et al. The role of epigenetics and long noncoding RNA MIAT in neuroendocrine prostate cancer. Epigenomics 8(5), 721-731 (2016).

3. Gupta RA, Shah N, Wang KC et al. Long non-coding RNA HOTAIR reprograms chromatin state to promote cancer metastasis. Nature 464(7291), 1071-1076 (2010).

4. Jain S, Thakkar N, Chhatai J, Pal Bhadra M, Bhadra U. Long non-coding RNA: functional agent for disease traits. RNA Biol. 14(5), 522-535 (2017). 
